\title{
Efficacy of Oxfendazole against Gastrointestinal Nematodes in Cattle and Buffaloes: A Clinical Study
}

\author{
Dilip Reddy G. ${ }^{1}$, Muralidhar Y. ${ }^{1}$, Surya Prasad V. ${ }^{2}$, Reddy G. $S^{3}$ \\ ${ }^{1}$ Assistant Professors, Department of Pharmacology \& Toxicology, College of Veterinary Science, Proddatur, \\ Andhra Pradesh, India - 516360. \\ ${ }^{2}$ Manager, Veterinary Services, ${ }^{3}$ Senior Vice President, ${ }^{2,3}$ Indian Immunologicals Limited, Rakshapuram, \\ Gachibowli, Hyderabad, Telangana, India - 500032
}

\begin{abstract}
In tropical and subtropical countries, the husbandry practices and the environment is ideal for parasite infestation and subsequent reduced productivity in cattle. The most important parasites are liver flukes, schistosomes, amphistomes, cestodes and GI tract nematodes. Oxfendazole is the sulphoxide metabolite of fenbendazole and responsible for that efficacy spectrum of fenbendazole. Oxfendazole reaches peak blood levels more slowly, thereby maintaining effective concentrations for a longer time, which increases the efficacy against immature and inhibited larvae. In the present study safety and efficacy of oxfendazole marketed by Indian Immunologicals Limited was studied in cattle and buffaloes. The efficacy was determined by measuring the Egg per gram (EPG) count in treated and control animals at different time intervals after administration of oxfendazole.No adverse events were observed in the animals. Oxfendazole was foundefficacious in reducing EPG by above $90 \%$ by 7 th day. The antiparasitic effect increased and continued till 21 st day indicating its efficacy in controlling the parasitic infestation.
\end{abstract}

Keywords: Buffaloes, cattle, efficacy, nematodes, oxfendazole,

\section{Introduction and background}

In mixed farming and extensive grazing systems practiced in tropical and subtropical countries, the environment is usually ideal for parasite infestation. This causes reduced productivity in terms of reduced weight gain, delayed and weak oestrus and lower calving rates and even greater impact is the lost production potential.The major effects are due to reduced feed intake and altered protein metabolism. Protein losses caused by parasites stimulate the synthesis of replacement proteins at the cost of energy and protein, which would normally be channeled into the production of meat and milk [1]. A large number of helminthic species are found in various animals in India, of which the most important are liver flukes, schistosomes, amphistomes, cestodes and gastrointestinal nematodes [2].

Oxfendazole is chemically methyl-5(6)-phenylsulfinyl-2-benzimidazole carbamate belongs to benzimidazole group of anthelmintics. It is the sulphoxide metabolite of fenbendazole and it is possible that efficacy spectrum of fenbendazole is due to its metabolite, oxfendazole. The pro-benzimidazole, febantel, when it is metabolized, also becomes fenbendazole, and finally oxfendazole. Oxfendazole administered orally to animals for treatment and control of gastro-intestinal roundworms, lungworms and tapeworms.Oxfendazole reaches peak blood levels more slowly from the intestinal tract than many of the older benzimidazoles, thereby maintaining effective concentrations for a longer time in both the serum and the intestinal tract. The persistence of effective concentrations increases the efficacy, particularly against immature and inhibited larvae.From various global efficacy studies the highest efficacy was seen at a dose of $5.0 \mathrm{mg}$ oxfendazole $/ \mathrm{kg}$ [3], [4], [5], [6].Toxicity, tolerance, and field safety studies were reported for oxfendazole. A product technical bulletin by European Medicines Agency [7]stated that no effects on the foetus were reported following maternal exposure to oxfendazole during pregnancy in animals. Hence a field clinical study is planned to investigate the safety and efficacy of oxfendazole marketed by Indian Immunologicals Limitedin cattle and buffaloes.

\subsection{Animals}

\section{Material and Methods}

All the animals enrolled in the study were from villages surrounding Proddatur. Not less than 40 numbers of animals for each species above 3 months of age with worm burden were enrolled in the study.

\subsubsection{Inclusion Criteria}

In order to minimize bias, only animals, which have worm infestation, as diagnosed by Eggs per Gram (EPG) count of around 150 were enrolled in each study group [1].

\subsubsection{Exclusion Criteria}

Animals underwent deworming treatment 12 weeks prior to the study and animals negative for any internal parasitic infestation were excluded. 


\subsection{Test Products and administration}

Oxfendazole boli manufactured by Indian Immunologicals Limited, Hyderabad (OXFENVET; Batch nos. OXF 2201 (2200 mg) were used in the study. Oxfendazole was administered orally@ 5mg/Kg body weight to all animals. The main parameters studied were safety of oxfendazole after administration in animals and during the study period and deworming efficacy (as indicated by reduced EPG count) of oxfendazole. The preferred method to evaluate the effectiveness in field studies is Egg count/larval identification [8].

\subsection{Study Design}

Enrolled animals of each species were randomly divided into two groups one control (10 no. of animals) and one treatment group (30 no. of animals) per species of animals based on faecal egg counts. The control (untreated) group was used to allow for monitoring of natural changes in egg counts during the test period.The controlanimals should equal a minimum of $25 \%$ of the test product treated animal numbers [9].

\subsection{Procedures}

At base line ( 2 days before enrolment), dung samples were screened and animals meeting the inclusion/exclusion criteria were enrolled in the study. The general health status of the animals was recorded.The dung samples were analyzed by various methods like direct smear examination, sedimentation technique and floatation technique. At Day 0, the enrolled animals were administered with oxfendazole according to the study design mentioned above. All the animals were monitored for adverse events during the study period.Dung samples were collected on 0th, $3^{\text {rd }}, 7^{\text {th }}, 14^{\text {th }}$ and $21^{\text {st }}$, days. All dung samples were processed and analyzed by McMaster egg counting technique for EPG counts at ourlaboratory for antiparasitic activity.

\subsection{Evaluation of efficacy}

The effectiveness is confirmed if it could reduce the concentration of eggs per gram of faeces (EPG) by more than 90 percent within 14 days of treatment.

\subsection{Statistical Analysis and Interpretation of data}

From EPG counts at different time intervals, the geometric mean and percentage reduction were calculated as geometric mean is a more appropriate estimate of central tendency for EPG counts and has less potential for misinterpretation than the arithmetic mean. In the case where some animals showing EPG counts=zero, then the geometric means were calculated by adding 1 to all values in the group and later subtracting 1 from mean.

The percentage reduction was calculated by the equation $=100(1-X t / X c)$

Where $X t$ is the mean egg count of the treated group and $X c$ is that of control group.

Efficacywas considered if the percentage reduction in egg counts wasmore than 90 percent.

\subsection{Safety evaluation}

Safety evaluation performed by observing and recording the adverse events observed during the first 30 minutes to 1 hour after drug administration. The events, which were observed or reported during the entire study period, were also evaluated for safety.

2.7.1 Adverse Events (AEs)

The animals were observed for any systemic reactions like excessive salivation, diarrhoea, fever, loss of appetite (Anorexia), restlessness and systemic adverse events etc.

\subsubsection{Serious Adverse Events (SAEs)}

The adverse event was considered as a SAE when the reaction resulted in death, was life-threatening, resulted in significant disability or incapacity or which resulted in permanent or prolonged signs in the animals treated with the drug under study.

\subsection{Animal Demographics}

\section{Results}

\subsubsection{Cattle:}

Total of 110 animal dung samples were screened for worm infestation out of which 57 were positive and 40 were included in study. The summary of demographics of cattle included in the study is presented below.

\begin{tabular}{|c|c|c|c|c|}
\hline Group & No of animals & Breed & $\begin{array}{c}\text { Mean Age (months) } \\
\text { (Range) }\end{array}$ & $\begin{array}{c}\text { Mean Weight (Kg) } \\
(\text { Range })\end{array}$ \\
\hline $\mathbf{1}$ & $30(\mathrm{M}-9 ;$ F-21) & HF cross-18; Jersey cr-5; Ongole-7 & $36.2(4-72)$ & $289.2(70-500)$ \\
\hline $\mathbf{2}$ & $10(\mathrm{M}-3 ;$ F-7) & HF cross-8; Ongole-2 & $32.4(6-60)$ & $262.0(90-450)$ \\
\hline
\end{tabular}

M-Male; F-Female; HF- Holstein Friesian 


\subsubsection{Buffaloes}

Total of 150 animal samples were screened for worm infestation out of which 58 were positive and 52 were included in study. The summary of demographics of buffaloes included in the study is presented below.

\begin{tabular}{|c|c|c|c|c|}
\hline Group & No of animals & Breed & Mean Age (months) (Range) & Mean Weight (Kg) (Range) \\
\hline 1 & $32(\mathrm{M}-7 ;$ F-25) & Graded Murrah & $33.0(4-72)$ & $274.4(90-500)$ \\
\cline { 5 - 6 } & $10(\mathrm{M}-3 ;$ F-7) & Graded Murrah & $31.5(5-72)$ & $288.5(75-500)$ \\
\hline
\end{tabular}

M-Male; F-Female

\subsection{Safety results}

There were no AEsor SAEs observed in cattle and buffaloes during the study.

\subsection{Efficacy}

From different screening methods performed, we could find eggs in floatation technique in majority of the positive samples indicating the presence of gastro intestinal nematodes in the region. The major nematode species eggs found in the study belongedto genera namely, Trichostrongyle, Ostertegia, Haemonchus, Cooperia, Paracooperia and Nematodirus. Hence, further analysis was performed by floatation technique to find out EPG count.

Summary of the EPG counts in different species of animals is presented below.

Mean (Geometric) EPG counts on different days in cattle

\begin{tabular}{|c|c|c|c|c|c|}
\hline Group & Day 0 & Day 3 & Day 7 & Day 14 & Day 21 \\
\hline 1 & 340.3 & 302.4 & $7.7(97.9 \%)$ & $2.8(99.2 \%)$ & $2.7(99.3 \%)$ \\
\hline 2 & 353.8 & 335.8 & 362.4 & \multicolumn{3}{c|}{358.7} & 374.8 \\
\hline \begin{tabular}{|c|c|c|c|c|c|}
\hline \\
\hline
\end{tabular} \\
Mean (Geometric) EPG counts on different days in Buffaloes \\
\hline 1 \\
\hline 2
\end{tabular}

Values in brackets indicate percent reduction in EPG compared to Control group

\section{Discussion and conclusion}

Oxfendazole, manufactured and marketed by Indian Immunologicals Limited, has not shown any adverse events in two species of the animals under study. The oxfendazole intreatment group was efficacious in reducing EPG by above $90 \%$ by $7^{\text {th }}$ day. The antiparasitic effect increased and continued till $21^{\text {st }}$ day indicating the efficacy of oxfendazole against nematodes in cattle and buffaloes.From the study it can be concluded that the Oxfendazole, manufactured and marketed by Indian Immunologicals Limited, Hyderabad is safe and effective in controlling gastrointestinal nematode parasites in cattle and buffaloes.

\section{References}

[1]. FAO working group on Parasite Resistance, Executive summary report. Downloaded from ftp://ftp.fao.org/docrep/fao/010/ag014e/ag014e02.pdf and Module 2 Helminths downloaded from ftp://ftp.fao.org/docrep/fao/010/ag014e/ag014e06.pdf, Rome, 2004

[2]. M C Agrawal, and P S Banerjee, Problems confronting helminthic diseases of domestic animals in India. Journal of Parasitic Diseases,31,2007, 3-13.

[3]. N Baker, R Fisk, J Miller, Anthelmintic Efficacy of Oxfendazole in Calves, American Journal of Veterinary Research, 39(8), 1978, 1258-61.

[4]. Miller J, Hembry F, Kearney M, Efficacy of oxfendazole against inhibited larvae of Ostertagia ostertagi acquired by calves in the spring American Journal of Veterinary Research, 49(7), 1988, 1007-1009.

[5]. R E Bradley, Efficacy of several formulations of oxfendazole administered to cattle as an anthelmintic,Agri-Practice, May-June, 1989,5 .

[6]. C E Couvillion, R C Herschler, C R Boyle, J A Hawkins, Efficacy of oxfendazole against inhibited larvae of Ostertagia ostertagi in naturally infected calves in the southern USA,TheVeterinary Record,124(7), 1989, 462-464.

[7]. European Medicines Agency, Veterinary Medicines and Inspections, EMEA/MRL/888/03-FINAL, June 2004, Committee for veterinary medicinal products, Oxfendazole(extrapolation to all ruminants), Summary Report (4)

[8]. VICH GL12, Guidance on Efficacy of anthelmintics: Specific recommendations for Bovines. International Cooperation on Harmonisation of Technical Requirements for Registration of Veterinary Medicinal Products (VICH), Brussels (Belgium), Guideline 12, 1999.

[9]. VICH GL7, Guidance on Efficacy of anthelmintics: General recommendations. International Cooperation on Harmonisation of Technical Requirements for Registration of Veterinary Medicinal Products (VICH), Brussels (Belgium), Guideline 7, 2000. 